\title{
Remote Sensing of Planar Ice Crystal Fall Attitudes
}

\author{
By Kenneth Sassen \\ Department of Meteorology, University of Utah, Salt Lake City, Utah 84112 \\ (Manuscript received 6 October 1979, in revised form 18 June 1980)
}

\begin{abstract}
The ability of planar ice crystals to assume horizontal orientations during fall is examined as a function of crystal diameter. After reviewing fall velocity measurements for the various planar crystal types, the fall attitude predictions from model experiments are compared to the results of photographic analyses of light pillar displays, an atmospheric optical phenomenon generated by near-horizontally aligned ice crystals. It is concluded that the prediction for stable fall in terms of Reynolds number $(R e)$ through the range $1.0<R e<$ 100 is generally valid in the atmosphere. It is further shown that planar crystals with $R e \approx$ 10 display the most stable fall attitudes, and that crystal diameters $>0.1-0.2 \mathrm{~mm}$ are required to generate the optical displays. It also appears that the distribution of ice crystal orientations from the horizontal plane is Gaussian in character owing to the effects of turbulence. The implications of these findings for other optical phenomena and the active remote sensing of cloud composition are discussed.
\end{abstract}

\section{Introduction}

Understanding the manner in which ice crystals fall through the atmosphere is of considerable importance to several areas of cloud physics research. The three-dimensional falling motion of an ice crystal may be quite complex, including particle rotation about the major axis, "fluttering" or spiralling oscillations about a preferred attitude, and changes in the fall velocity (i.e., the vertical component of the falling motion) related to fall attitude variations. Knowledge of this falling behavior is prerequisit to understanding the growth of precipitation particles through the ice crystal aggregational and crystal-droplet accretional growth mechanisms. Moreover, the ability of ice crystals to maintain certain orientations has important implications for modelling atmospheric optical phenomena, and also for interpreting backscattered laser and microwave signals under some conditions (Platt, 1978; Hendry et al., 1976).

Two basic approaches have been used to determine the fall attitudes of ice crystals in the atmosphere. The first method involves the simulation of the falling behavior of ice crystal-like models in dense fluids (e.g., water or glycerin), with the findings generalized to the atmospheric case through theory derived from fluid mechanics. The second method relies on photographic observations of the falling behavior of particles grown in the laboratory or sampled within natural clouds from mountain-based observatories. In addition to such direct observations, much useful information has also been gained indirectly from ground-based and airborne studies of the shape and riming properties of ice crystals (see, e.g., Ono, 1969).

In the present study, a different approach is used to derive information describing the fall attitude instabilities experienced by crystals in the atmosphere. This is accomplished through a passive remote sensing technique involving the analysis of photographs of light pillars, a variety of man-made optical phenomenon which appears at night as vertical columns of light above street lamps and other bright sources during light snowfall or ice fog. Such displays are caused by light which is simply reflected off the faces of nearhorizontally aligned ice crystals present between the source and the observer. Since light will be reflected toward the observer only when striking the crystal face at normal incidence, it follows that the apparent width of the pillar is controlled by the maximum wobble angle of the crystals, while the variation in the intensity of light across 
the pillar is due to the distribution of crystal orientations from the horizontal plane. Thus, it can be seen that the analysis of light pillar photographs provides unique information describing the fall attitudes of a population of ice crystals, as will be illustrated here. Our findings are evaluated with regard to the type and size of the ice crystals concurrently collected at the ground, and the results are compared to available predictions based on model experiments. As described in Sassen (1980), which gives additional information on these occurrences, the ice crystals sampled belonged almost exclusively to the planar crystal habits. Therefore, we confine our review of earlier findings below to results dealing with planar ice crystals.

\section{The falling behavior of planar crystals; a review}

The most stable, or "preferred" orientation of a falling disk-like particle is regarded as that position in which the particle falls vertically at constant speed with the large basal faces aligned in the horizontal plane (i.e., orthogonal to the direction of motion). The particle size range over which this stable fall attitude is generally maintained can be delineated through the use of the dimensionless Reynolds number, $R e$, as defined by

$$
R e=v d / \nu,
$$

where $v$ is the fall velocity of a particle with diameter $d$, and $\nu$ is the kinematic viscosity of the fluid. The $R e$ regime for stable fall depends on particle shape, and these regions must be found through experimental means for irregularlyshaped particles. The utility of this parameter arises from the belief that the relation between Reynolds number and the falling behavior of a particle of specified shape is largely independent of the actual experimental set-up chosen to simulate atmospheric conditions. Hence, "model" crystal forms and fluid media convenient for study in a laboratory tank can be used and the results applied to a situation in the atmosphere with similar Reynolds number.

On the basis of such model experiments, it has been found that thin disks (with diameter-tothickness ratios of $\leq 0.1$ ) tend to generally assume a horizontal attitude in a quiescent fluid through the range of $1<R e<100$ (see, e.g., Willmarth et al., 1964). For smaller $R e$, the falling disks tend to maintain the orientation that they displayed upon being released into the fluid. For larger
$R e$, the fluid flow around the particle and in its wake becomes unstable, causing the disk to undergo pitching oscillations or spiralling motions. Pitching oscillations are also observed to occur for $1<\operatorname{Re}<100$ as the disk initially adjusts to the horizontal position or as inhomogeneities in the fluid are encountered, but these oscillations rapidly dampen out for most Reynolds numbers in this range. Little damping, on the other hand, is observed as $R e$ approaches 100 .

In order to interpret these findings in terms of the fall attitudes of planar ice crystals in the atmosphere, what is foremost required is a knowledge of the fall velocities of these particles as a function of diameter (i.e., maximum crystal dimension). Although the determination of ice particle fall velocity has received attention from numerous investigators, until recently no comprehensive measurements were available. From the studies of Kajikawa (1971, 1972, 1973, 1975) it is now possible to specify the fall velocity of the various planar ice crystal types through a large range of crystal sizes. Figure 1 compiles this information for the five planar ice crystal habits indicated. Note that given for reference in this figure as the dashed curve is the fall velocity versus diameter relationship for cloud droplets, as computed from Stokes' Law for $d<0.1 \mathrm{~mm}$ and taken from the measurements of Gunn and Kinzer (1949) for $d>0.1 \mathrm{~mm}$.

The data shown in Figure 1 represent the fall velocities of particles at a pressure and temperature of $1,000 \mathrm{mb}$ and $-15^{\circ} \mathrm{C}$. With the exception of the experimental data for plates and branched-plates for $d<0.1 \mathrm{~mm}$ from Kajikawa (1973), all solid lines have been taken from theoretical predictions based on model experiments. These curves, however, generally show good agreement with the measurements reported by Kajikawa. The dashed lines represent our extrapolations of the data in diameter intervals where experimental verifications are lacking. The branching appearance of the ice crystal $v$-d relationships can be justified with the knowledge that minute ice crystals growing in the planar crystal regime do not appear to initially display much differentiation in shape, while the appearance of the branched crystal forms is related to ventilation effects in relatively rapidly falling plates. Ono (1969), for example, noted the onset of dendritic growth in natural clouds for plates on the order of $0.2-0.3 \mathrm{~mm}$ diameter. It is also interesting to note that plate crystals of $<25 \mu \mathrm{m}$ diameter display larger fall speeds than water 


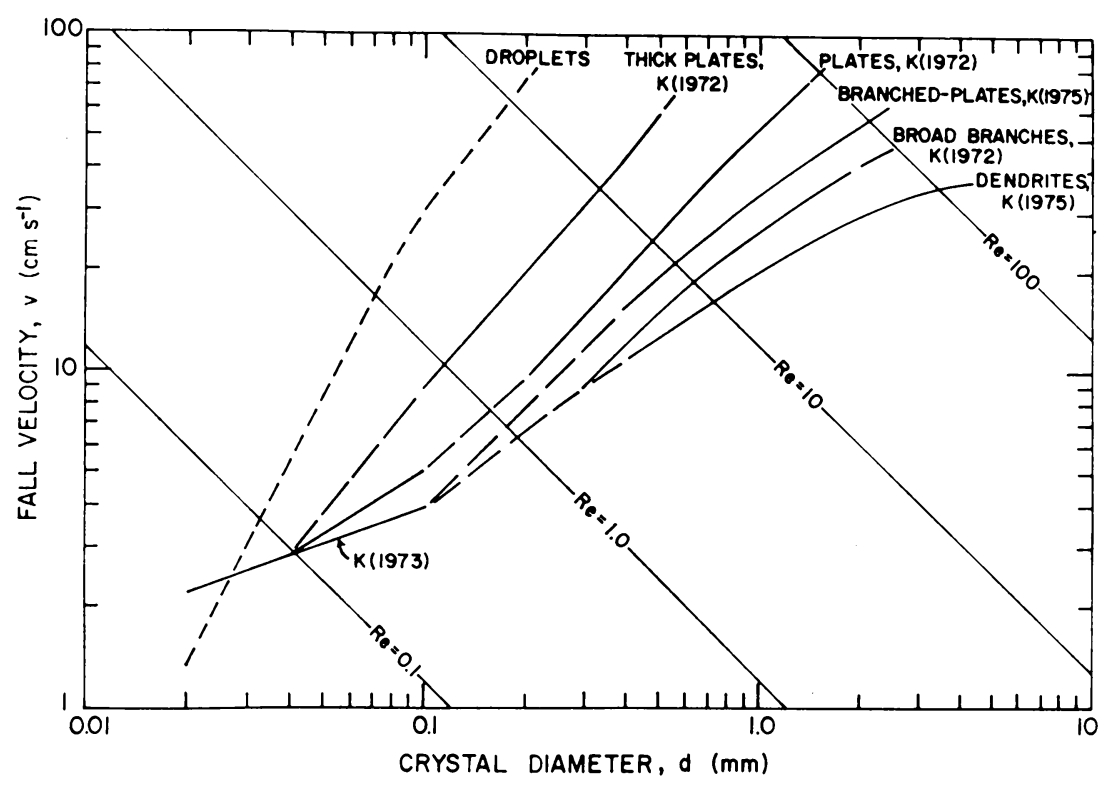

Fig. 1 A compilation of the fall velocity versus crystal diameter relationships for the five planar ice crystal types derived from the studies of Kajikawa (K), with the relationship for water droplets also shown (dashed line). Slanted lines depict constant Reynolds numbers $(R e)$.

spheres of the same diameter, a finding that may be attributed to a vertical edge-on falling motion for plates of this size (see Kajikawa, 1973).

The lines of constant $R e$ values shown as thin slanted lines in Figure 1, also calculated for $1,000 \mathrm{mb}$ and $-15^{\circ} \mathrm{C}$, can now be used to determine the planar crystal size ranges corresponding to the stable fall regime predicted from the model experiments. We find that the thin plate varieties should begin to assume their preferred orientations at $0.15-0.2 \mathrm{~mm}$ diameters, whereas $R e=100$ is reached for plates and dendrites of about $1.5 \mathrm{~mm}$ and $3.5 \mathrm{~mm}$ diameter, respectively. Thick plates with their greater mass-to-diameter ratio should achieve stable descent at $0.1 \mathrm{~mm}$. While these predictions correspond to the atmospheric situation near sea level pressure, it should be recalled that $R e$ is a function of fluid viscosity, or atmospheric density. To illustrate the effect of air density, given in Figure 2 is the dependence of the $R e-d$ relationship for plate crystals as a function of atmospheric pressure.

The curves shown in Figure 2 for pressures between 1000 and $300 \mathrm{mb}$ (in $100 \mathrm{mb}$ intervals) have been computed using the technique described by Kajikawa $(1971,1972)$. It is evident from the these results that the $R e-d$ relationship for plates is not greatly sensitive to pressure in the troposphere. That the effect is not as great

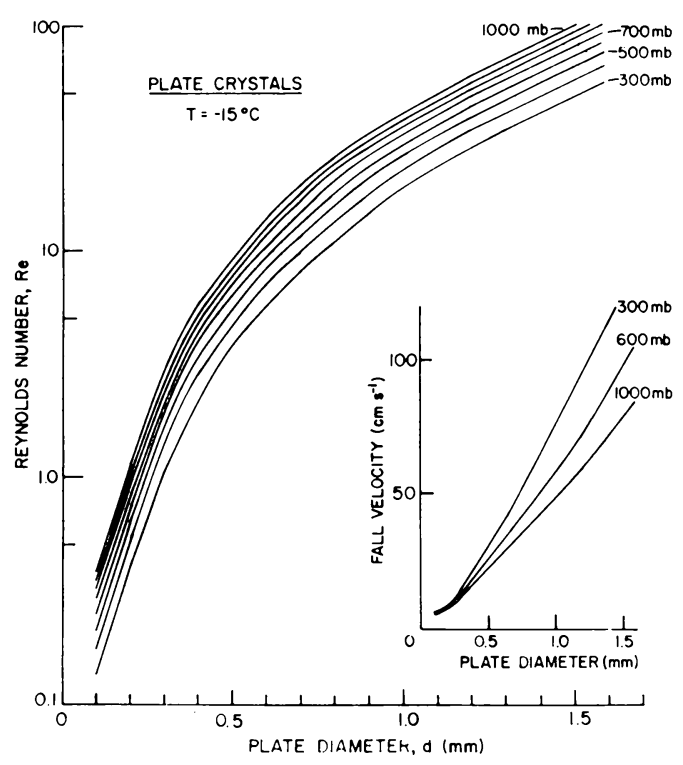

Fig. 2 The variations in Reynolds number with plate crystal diameter as a function of atmospheric pressure in $100 \mathrm{mb}$ intervals. Insert shows dependence of fall velocity relationship on atmospheric pressure.

as might be expected from the decrease in air density with height is a result of the counteracting increase in particle fall velocities (see 
insert in Figure 2). Thus, although the light pillar observations were conducted at $2.2 \mathrm{~km}$ above sea level with a typical surface pressure of $775 \mathrm{mb}$, our findings concerning fall attitudes may be applied to the crystal size predictions for sea level conditions discussed above without serious error.

\section{Experimental results}

Light pillar photographs and ice crystal samples were collected on a total of 15 occasions through the three-year study period, while the composition of snowfall which occurred under similar conditions but which failed to generate light pillars was also frequently examined. The types and modal diameters of the planar crystals responsible for the optical displays can be seen from an examination of Figure 3, where the various symbols identify the dominant crystal types observed in each case. Note that it was only in one case that prismatic ice crystals were found to be responsible for light pillar formation (see Sassen, 1980).

The range of $R e$ corresponding to the diameters of the crystals causing the displays can be seen to be in agreement with our expectations based on the model experiments for thin disks. Although the modal diameters of the smallest plate and thick plate crystals observed to produce pillars correspond to $\operatorname{Re}<1$, in both cases numerous larger crystals with diameters up to $0.15 \mathrm{~mm}$ $(R e \approx 1)$ were also present. When crystals smaller than $0.15 \mathrm{~mm}$ diameter were sampled, no pillars were observed. Similarly, the upper limit of crystal modal diameters corresponded to $R e \approx$ 100 . The $R e$ regions where no optical phenomena were observed are marked "No Pillars" in the figure, while also included are some comments on the amount of angular broadening of the pillars with height. This kind of information indicates the degree to which the particles were successful at maintaining perfect horizontal orientations, as examined in greater detail below.

Our understanding of the scattering geometry involved in light pillar formation predicts that the angle of the maximum crystal wobble from the horizontal plane can be estimated from the measurement of the pillar half-width angle recorded on the photographic film. In dealing with photographic images, however, care must be taken to ensure the uniformity of results from such analyses. In particular, in measuring the widths of a light pillar image the background sky brightness has an impact on deciding where

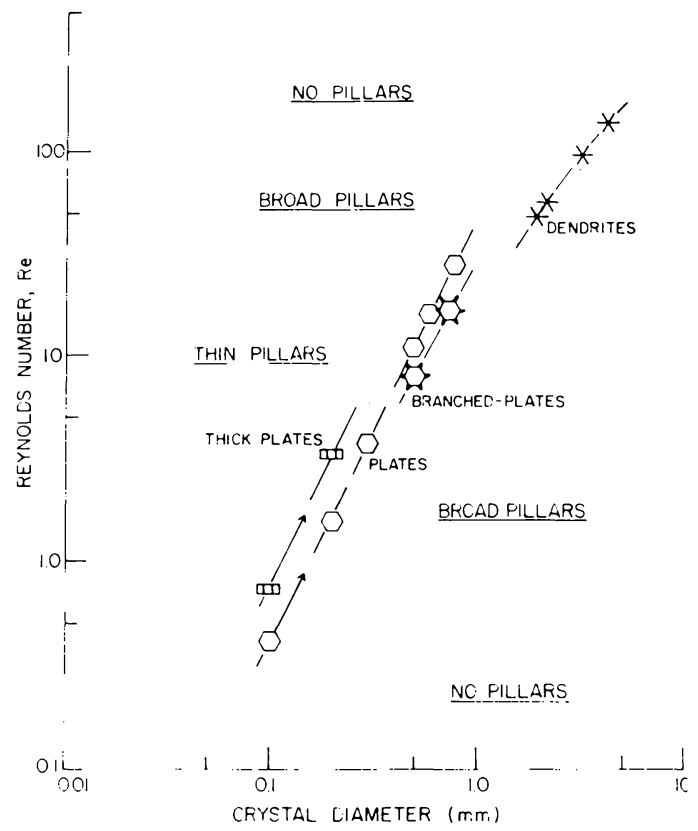

Fig. 3 The modal diameters and Reynolds numbers of the indicated ice crystal types found to be responsible for the formation of light pillars. In agreement with model experiments for thin disks, light pillars are observed only through the approximate range of $1<\operatorname{Re}<100$.

the pillar margins are to be located. Fortunately, most of the light pillar displays occurred during very light intensity snowfalls of single ice crystals and so were accompanied by excellent visibility, while the photographs were taken from a remote rural site where glare from urban light pollution was minimal in many viewing directions. Hence, the pillars generated by a group of intense light sources $\sim 0.5-1.0 \mathrm{~km}$ distant were selected for detailed study, and the lower, brightest pillar margins were traced by projecting the negatives on paper to obtain estimates of the pillar width angles for about two-thirds of the cases studied which were suitable for this analysis. The use of these procedures should provide for fairly uniform results under the special conditions associated with the formation of light pillars in an optically thin and anisotropic scattering medium, in which multiple scattering and non-specular reflection contributions can be expected to be relatively unimportant. Note, however, that all pillar half-width angles may be slightly exaggerated due to the finite size of the light sources 


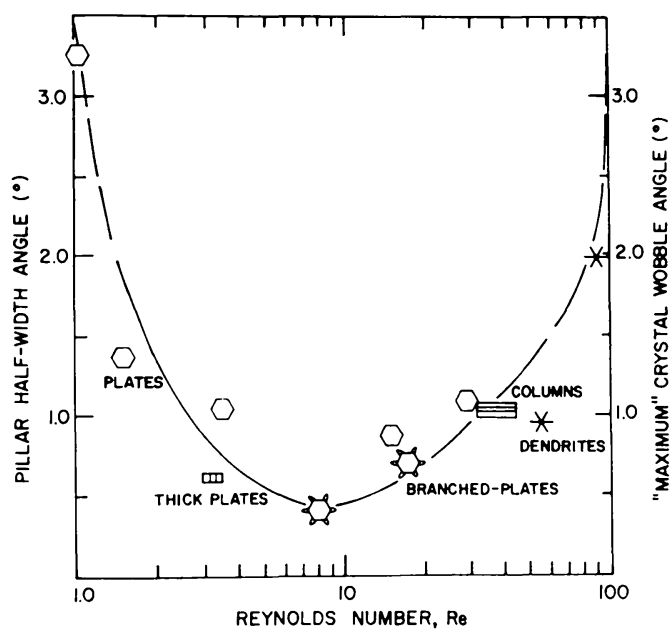

Fig. 4 Average light pillar half-width angles derived from photographs plotted as a function of the modal crystal diameter Reynolds number. The pillar width measurements provide an indication of the "maximum" crystal wobble from the horizontal plane, and predict that crystals with $R e \approx 10$ display the most stable fall attitudes.

(the apparent size of the solar disk produces a similar effect), while the widths of broad and faint light pillars were difficult to measure in this way and were probably underestimated.

The results of such an analysis are shown in Figure 4, where the averaged half-width angle is given as a function of the Reynolds number determined from the modal diameter of the dominant crystal type present. Despite the fact that the pillar widths may be expected to be influenced by the size distributions of the crystals present in each case, the results shown in Figure 4 reveal that the "maximum" crystal deviations from the horizontal plane are a function of $R e$. It can be concluded that crystals with $R e \approx 10$ display the most perfect orientations, and that with increasing or decreasing $R e$ the particles tend to deviate from this position to an increasingly greater extent. The absence of light pillars for $R e$ just below 1 or greater than 100 must then be regarded as representing a condition in which the particles fluttered too widely from the horizontal plane to produce a visible light pillar, and do not therefore indicate random orientations. Note also that the pillar half-width angle for $1.5 \mathrm{~mm}$ modal length columnar crystals is in agreement with the results for planar crystals.
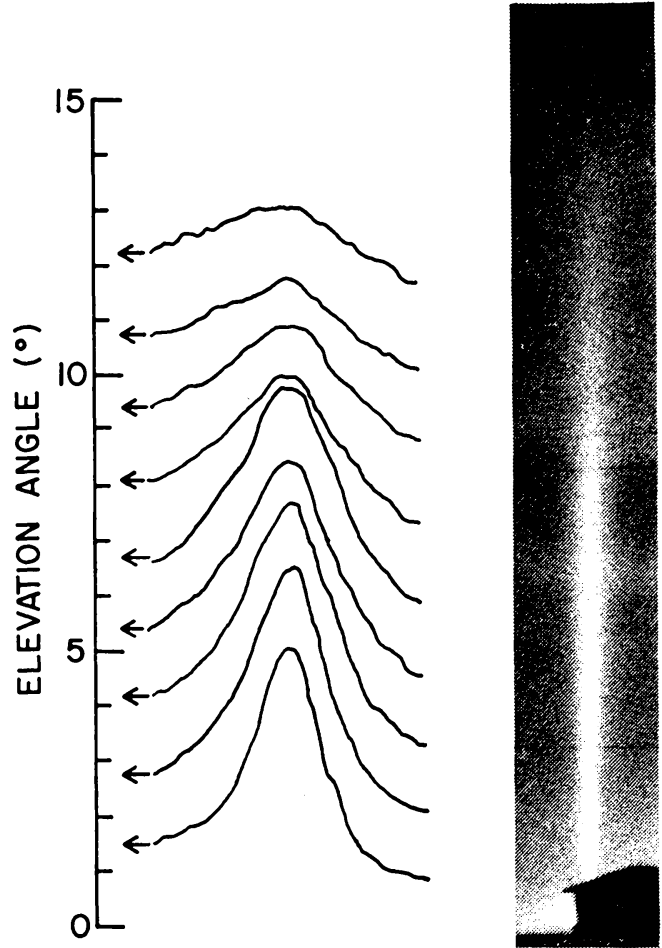

Fig. 5 The results of a series of microdensitometer line-scans at $\sim 1.3^{\circ}$ elevation angle intervals across the light pillar shown on the right-hand side of the figure. In each scan the reflected light intensity increases upward, demonstrating that the ice crystal orientations causing the Gaussian intensity profiles are distributed normally from the horizontal plane.

In Figure 5 we present the results of a microdensitometer analysis of a typical photographic image of a light pillar generated in this case by plate and thick plate crystals with a narrow size distribution about the $0.2 \mathrm{~mm}$ modal diameter. For comparison, given on the right-hand side of the figure is the pillar photograph enlarged to approximately the same scale as the elevation angles of the horizontal line-scans which are indicated by the arrows at the bottom of each scan. Note that the optical density plots were obtained by scanning the instrument across the photographic negative at very high resolution, such that the horizontal distance is greatly expanded. The relative pillar widths can be seen to gradually increase with increasing elevation angle owing to the effect of the divergent light source on the light pillar scattering geometry. However, 
the line-scans reveal that the intensity variations across the horizontal pillar sections resemble quite closely Gaussian curves, demonstrating that the orientations of the ice crystals contributing to the display are distributed normally from the horizontal plane. When the spreading of the Gaussian pillar profiles is normalized with respect to the clevation angle, the resultant intensity curves are generally in close agreement, indicating little change in the crystal fall attitudes with height in this case.

On the basis of these findings, we can in general characterize the distribution of crystal orientations in the atmosphere as Gaussian in nature, with the "widths" of the distributions being a function of crystal Reynolds number as shown approximately in Figure 4. Note, though, that the pillar half-width angle measurements of Figure 4 cannot then represent the actual "maximum" crystal wobble angles, but it may be assumed as an approximation that the measured pillar width angles correspond to two standard deviations from the pillar intensity peak for the purpose of determining the range of crystal wobble angles that are recorded with the photographic method under favorable backlighting conditions.

\section{Summary and discussions}

Through model experiments and the application of fluid dynamics theory, we may predict the falling motions of ice crystals in the atmosphere, provided that we have knowledge of the falling velocities of the various kinds of ice crystals. Fortunately, these data are now largely available as a function of crystal size (see Figure 1 for planar crystal $v-d$ relationships). The results of our analysis of light pillars, a type of optical phenomenon which requires the presence of nearhorizontally aligned ice crystals, yields agreement with the stable fall predictions from the model experiments-that is, light pillars were observed only for ice crystals within the approximate range of $1.0<\operatorname{Re}<100$. However, we have found additional evidence that the ability of such crystals to maintain horizontal orientations is a function of the Reynolds number, with crystals of $R e \approx 10$ showing the most perfect alignment. That the model experiments do not predict the exact falling behavior of ice crystals may be regarded as a consequence of the differences in the fluid media of the simulated and real atmospheres.

In model experiments performed in a quiescent laboratory tank the only destabilizing force en- countered is that related to the shedding of turbulent vortices in the particles wake, as can be visualized by coating the model with a suitable dye (sce Willmarth et al., 1964). This mechanism accounts for the pitching and translational oscillations for ice crystals with $R e>100$, and eventually leads to tumbling motions for very large $R e$. In the real atmosphere, however, additional destabilizing forces must be considered. For very small particles Brownian motions would constantly re-align the crystals, resulting in random orientations. In addition, unlike the quiescent laboratory fluid media, the atmosphere is disturbed by various scales of turbulent air motions. Although the influence of turbulence on ice crystal motions is still poorly understood, we may expect small-scale turbulent air motions to displace the crystals from their initial orientations. It has been shown from the model experiments that the time required for the particle to adjust to such a fall attitude perturbation depends on the Reynolds number. Therefore, it seems reasonable to account for the variations in fall attitudes shown in Figure 4 on the basis of the response of the crystals to turbulence-induced fall attitude perturbations. Turbulence can also be expected to aid in the reorientation of crystals with $R e<$ 1.0 into random positions, and to increase the instability of the pitching oscillations for $R e>$ 100.

This situation, in which at least a portion of the population of crystals is in the process of correcting for turbulence-induced fall attitude perturbations, can be physically viewed as leading to the Gaussian distribution of crystal deviations from the horizontal plane that we have inferred from our microdensitometer analyses. Hence, such measurements contain information which may be used to characterize the turbulent air motions present in the atmosphere.

With this knowledge of the falling motions of atmospheric ice crystals, several research areas which require a knowledge of ice crystal falling behavior may be treated with more certainty. In addition to contributing to our understanding of basic cloud microphysical processes, such information is clearly pertinent to the field of meteorological optics. Atmospheric optical phenomena due to ice crystals have traditionally been divided into two broad categories based on the ability of ice crystals to orient uniformly in space. The presence of the common haloes is most easily explained for the case of randomly oriented ice crystals, while uniformly oriented crystal popu- 
lations of various shapes are required to account for most other displays. Our findings indicate that ice crystals with maximum dimensions greater than about $0.1-0.2 \mathrm{~mm}$ would generally be required for the optical displays of the latter category. Crystals smaller than $\sim 0.1 \mathrm{~mm}$, however, would not necessarily display completely random orientations.

Recent calculations by Fraser (1979) predict a state of complete random orientation for an oblate ellipsoid model of a thin plate for diameters $<\sim 10 \mu \mathrm{m}$, with particles up to $\sim 40 \mu \mathrm{m}$ still exhibiting deviations from the stable fall position resultant from Brownian motion. On the other hand, laboratory scattering measurements (Sassen and Liou, 1979) indicate that crystals $>20 \mu \mathrm{m}$ diameter are required in order to generate even weak haloes. Hence, on the basis of this information, it can be inferred that ice crystal populations which are in a state of complete random orientation can not be responsible for halo formation. Although it is possible that ice crystals between about $20-100 \mu \mathrm{m}$ maximum dimensions may be sufficiently disturbed by turbulence to produce rather uniform-appearing haloes, an altcrnate explanation for haloes can be found in terms of the crystal types frequently encountered in cirrus clouds. It is well known that such clouds are often composed of ice crystal clusters in the form of radiating groups of column or bullet crystals (see e.g., Heymsfield and Knollenberg, 1972). Since the individual crystal elements of such clusters can be considered to be randomly arrayed, the conditions required for halo formation may be satisfied regardless of the overall particle size. It should be pointed out that recent airborne probing of a cirrus cloud layer which produced halo phenomena found such spatial crystals to be predominantly present (Glass and Varley, 1978).

Finally, we should like to briefly consider another application for our results. Active remote sensing techniques using polarized laser or microwave beams have in recent years shown considerable promise for the inference of cloud composition. Populations of uniformly arrayed ice crystals appear to produce a medium with peculiar scattering properties, providing the opportunity to remotely determine the composition of such clouds. Platt (1978), for example, has shown that a significant amount of information can be recovered from lidar observations of clouds containing horizontally-oriented plate crystals. Our findings, particularly with regard to the
Gaussian distribution of crystal fall attitude deviations, can provide the basis for more precise methods of obtaining cloud composition data under these unique conditions. It is also clear that remote sensing observations would have to be performed within a few degrees of the vertical direction in order to have a high probability of detecting the specular reflections from oriented plate crystal populations.

\section{Acknowledgements}

The author received support during the preparation of this paper and for recent microdensitometer studies from the Air Force Office of Scientific Research, under Contract F4962079-C-0198.

\section{References}

Fraser, A. B., 1979: What size of ice crystals causes the haloes? J. Opt. Soc. Am., 69, 1112-1118.

Glass, M. and D. J. Varley, 1978: Observations of cirrus particle characteristics occurring with halos. Preprints Conf. Cloud Physics and Atmospheric Electricity, Issaquah, Amer. Meteor. Soc., 126128.

Gunn, R. and G. D. Kinzer, 1949: The terminal velocity of fall for water drops in stagnant air. J. Meteor., 6, 243-248.

Hendry, A., G. C. McCormick and B. L. Barge, 1976: The degree of common orientation of hydrometeors observed by polarization diversity radars. J. Appl. Meteor., 15, 633-640.

Heymsfield, A. J. and R. G. Knollenberg, 1972: Properties of cirrus generating cells. J. Atmos. Sci., 29, 1358-1366.

Kajikawa, M., 1971: A model experimental study on the falling velocity of ice crystals. J. Meteor. Soc. Japan, 49, 367-375.

_ 1972: Measurement of falling velocity of individual snow crystals. J. Meteor. Soc. Japan, 50, 577-584.

- 1973: Laboratory measurement of falling velocity of individual ice crystals. J. Meteor. Soc. Japan, 51, 263-272.

, 1975: Experimental formula of falling velocity of snow crystals. J. Meteor. Soc. Japan, 53, 267-275.

Ono, A., 1969: The shape and riming properties of ice crystals in natural clouds. J. Atmos. Sci., 26, 138-147.

Platt, C. M. R., 1978: Lidar backscattering from horizontal ice crystal plates. J. Appl. Meteor., 17, $482-488$.

Sassen, K., 1980: Light pillar climatology and microphysics at Laramie, Wyoming. Weatherwise, 33, (in press).

and K. N. Liou, 1979: Scattering of 
polarized laser light by water droplet, mixed phase and ice crystal clouds: I. Angular scattering patterns. J. Atmos. Sci., 36, 838-851.
Willmarth, W. W., N. E. Hawk and R. L. Harvey, 1964: Steady and unsteady motions and wakes of freely falling disks. Phys. Fluids, 7, 197-208.

\section{板状水晶の大気中での落下姿勢に関するリモートセンシング}

\section{Kenneth Sassen}

University of Utah, U.S.A.

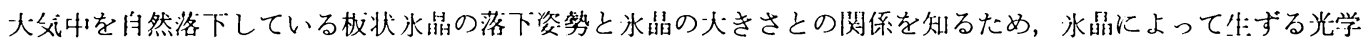

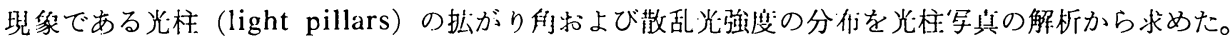

その結果，レイノルズ数（Re）にして $1.0<R e<100$ の籁囲の氷晶は，基底面（basal plane）を落下力向に

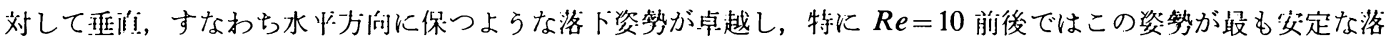
下姿势であることが判った。

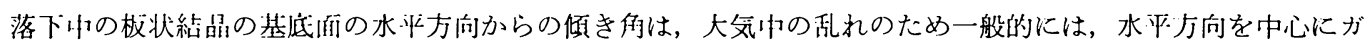
ウス分们をしている。

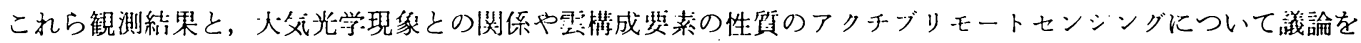
行った。 